\title{
Drag on an Ellipsoid Particle in Free-Molecular Plasma Flow
}

\author{
Ming Li, ${ }^{1,2}$ Yunming Chen, ${ }^{1}$ and D. B. Graves ${ }^{2}$ \\ Received January 8, 1996; revised June 17, 1996
}

Based on the analysis of molecular gas dynamics, the drag and moment acting on an ellipsoid particle of revolution $X^{2} / a^{2}+Y^{2} / a^{2}+Z^{2} / c^{2}=1$, as an example of nonspherical particles, are studied under the condition of free-molecular plasma flow with thin plasma sheaths. A nonzero moment which causes nonspherical particle self-oscillation and self-rotation around its own axis in the plasma flow-similar to the pitching moment in aerodynamics-is discovered for the first time. When the ratio of axis length $\mathrm{c} / \mathrm{a}$ is unity, the moment is zero and the drag formula are reduced to the well-known results of spherical particles. The effects of the particleplasma relative velocity, the plasma temperature, and the particle materials on the drag and moment are also investigated.

KEY WORDS: Drag; ellipsoid particles; self-oscillating or self-rotating; rarefied plasma flow.

\section{INTRODUCTION}

Thermal plasmas are extensively used in the processing of particulate matter, such as the synthesizing and spheroidizing of fine or ultrafine powder materials, thermal plasma sputtering etc., and thus it is essential to study the heat transferring to and the force acting on the particle in plasmas. ${ }^{(1,2)}$ Although intensive efforts have been devoted by many researchers in the recent years, ${ }^{(3-15)}$ the effects of particle shape on the particle charging, heat transfer, and force are still little known and need further studies. ${ }^{(1)}$

During the past decade, the floating potential of a particle in plasmas and the heat transfer to the particle from plasmas were extensively investigated, including many factors such as the effects of particle materials (conductors and insulators) ${ }^{(6)}$ the effects of thick plasma sheaths, ${ }^{(7)}$ thermal electron emissions, ${ }^{(8-11)}$ particle radiations, ${ }^{(9)}$ and the effects of positive floating potential. ${ }^{(12)}$ Recently, heat transfer to an ellipsoid particle of revolution

'Institute of Mechanics, Chinese Academy of Sciences, Beijing 100080, P.R. China.

${ }^{2}$ Department of Chemical Engineering, University of California at Berkeley, Berkeley, California 94720 . 


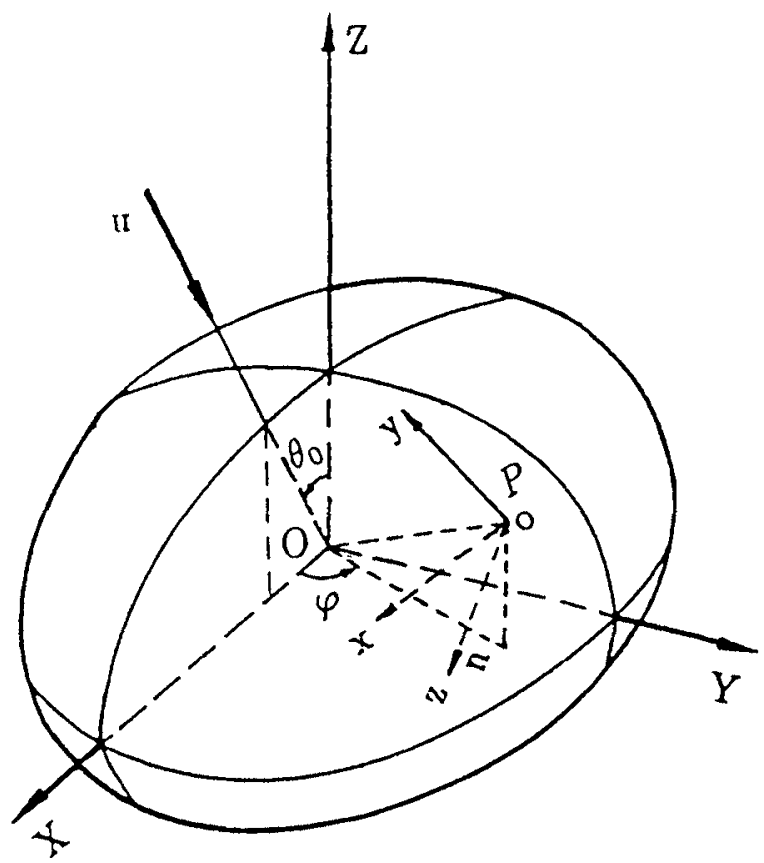

Fig. 1. Schematic of the coordinates.

was studied by Gnedovets et al. ${ }^{(13)}$ and the authors. ${ }^{(14)}$ An equivalent sphere with the same surface area as the ellipsoid was suggested by the authors ${ }^{(14)}$ to calculate the heat transfer to the nonspherical particle from rarefied plasma.

In the present paper, the method of molecular gas dynamics is adopted to study the drag and rotating moment acting on an ellipsoid particle $\mathbf{X}^{2} / \mathbf{a}^{2}+\mathbf{Y}^{2} / \mathbf{a}^{2}+\mathbf{Z}^{2} / \mathbf{c}^{2}=1$, as shown in Fig. 1, under conditions of thin plasma sheath and free molecular flow. The effects of the plasma temperature, the relative velocity between plasmas and particles, and the ratio of $c / a$ on the drag and moment are investigated.

\section{ANALYTICAL MODEL}

\subsection{Basic Assumptions}

When a particle is immersed in plasmas, a great number of collisions with electrons, positive ions, and neutral atoms result in the negative charging of the particle, and the momentum and energy transfer between the 
particle and the plasma species. Generally, the following assumptions are adopted. $^{(3-5.12-15)}$

The characteristic size of the particle is much less than the average free path length but much larger than the Debye length of each plasma species.

The plasma consists of atoms, single ionized ions, and electrons. The temperature for heavy species (atoms and ions) may be different from that of electrons.

All electrons colliding with the particle are absorbed on the particle surface, but atoms and the recombined ions arriving at the particle all undergo complete diffuse reflection at the particle surface with the same temperature as the particle.

The exchange of momentum between plasmas and particles is considered under the condition of steady floating potential, i.e., the effect of initial charging process is neglected.

The thermal electron emission, radiation and evaporation of the particle are all neglected when the particle surface temperature is not high enough.

\subsection{The Floating Potential on the Particle Surface}

The coordinate systems OXYZ, as shown in Fig. 1, is fixed on the ellipsoid particle of revolution $X^{2} / \mathbf{a}^{2}+\mathbf{Y}^{2} / \mathbf{a}^{2}+\mathbf{Z}^{2} / \mathbf{c}^{2}=1$ while oxyz relates to the position on the particle surface, $\mathbf{o x}$, oy corresponds to the two perpendicular tangents, and $\mathbf{o z}$ is in the inner normal direction of the particle surface. For a point $P$ at the particle surface $P(X, Y, Z)=(a \sin \theta \cos \varphi$, $a \sin \theta \sin \varphi, c \cos \theta$ ), the corresponding direction cosines of tangents $\mathbf{o x}, \mathbf{o y}$ and the normal $n$ are

$$
\begin{aligned}
& \cos \alpha_{1}=-\sin \varphi, \quad \cos \beta_{1}=\cos \varphi, \quad \cos \gamma_{1}=0 ; \\
& \cos \alpha_{2}=\cos \theta \cos \varphi / \sqrt{\cos ^{2} \theta+(c / a)^{2} \sin ^{2} \theta}, \\
& \cos \beta_{2}=\cos \theta \sin \varphi / \sqrt{\cos ^{2} \theta+(c / a)^{2} \sin ^{2} \theta}, \\
& \cos \gamma_{2}=-(c / a) \sin \theta / \sqrt{\cos ^{2} \theta+(c / a)^{2} \sin ^{2} \theta} ; \\
& \cos \alpha=-(c / a) \sin \theta \cos \varphi / \sqrt{\cos ^{2} \theta+(c / a)^{2} \sin ^{2} \theta}, \\
& \cos \beta=-(c / a) \sin \theta \sin \varphi / \sqrt{\cos ^{2} \theta+(c / a)^{2} \sin ^{2} \theta}, \\
& \cos \gamma=-\cos \theta / \sqrt{\cos ^{2} \theta+(c / a)^{2} \sin ^{2} \theta} .
\end{aligned}
$$


The plasma oncoming flow is oriented in the plane of $\mathrm{XOZ}$ and the relative velocity between the plasma and the particle is $\vec{u}=-u \sin \theta_{0} \vec{i}_{X}-u \cos \theta_{0}$ $\vec{i}_{Z}$

The velocity distribution functions for each species incident on the particle obey Maxwellian distribution. ${ }^{(3-15)}$

$$
f_{j}^{-}=\frac{n_{j}}{\left(2 \pi k_{B} T_{j} / m_{j}\right)^{3 / 2}} \exp \left[-\frac{\left(v_{x}-u_{x}\right)^{2}+\left(v_{y}-u_{y}\right)^{2}+\left(v_{z}-u_{z}\right)^{2}}{2 k_{B} T_{j} / m_{j}}\right]
$$

where

$$
\left(\begin{array}{l}
u_{x} \\
u_{y} \\
u_{z}
\end{array}\right)=u\left(\begin{array}{l}
B_{x} \\
B_{y} \\
B_{z}
\end{array}\right)=u\left(\begin{array}{lll}
\cos \alpha_{1} & \cos \beta_{1} & 0 \\
\cos \alpha_{2} & \cos \beta_{2} & \cos \gamma_{2} \\
\cos \alpha & \cos \beta & \cos \gamma
\end{array}\right)\left(\begin{array}{c}
-\sin \theta_{0} \\
0 \\
-\cos \theta_{0}
\end{array}\right)
$$

the subscript $j$ denotes electrons $(j=e)$, ions $(j=i)$, and atoms $(j=a), n, T$, and $m$ are the number density, temperature, and mass, respectively, $k_{B}$ is the Boltzmann constant, and $v_{x}, v_{y}, v_{z}$ are the thermal velocity of each plasma species.

The flux of each plasma species incident on the particle surface can be calculated by

$$
\psi_{j}=\int_{-\infty}^{\infty} d v_{x} \int_{-\infty}^{\infty} d v_{y} \int_{A}^{\infty} v_{z} f_{j}^{-} d v_{z}
$$

The lower integration limit $A$ is zero for atoms and ions $(j=a, i)$ while it is $\sqrt{-2 e \phi_{f} / m_{e}}$ for electrons $(j=e)$ since any atom and ion with positive velocity can reach the particle while any electron with $v_{z}$ less than $\sqrt{-2 e \phi_{f} / m_{e}}$ cannot arrive due to the barrier of the negative floating potential $\phi_{f}$. Briefly, the flux to the particle surface for each plasma species is

$$
\begin{aligned}
& \psi_{j}=\frac{1}{4} n_{j} \bar{v}_{j}\left\{\exp \left(-S_{h}^{2} B_{z}^{2}\right)+\sqrt{\pi} S_{h} B_{z}\left[1+\operatorname{erf}\left(S_{h} B_{z}\right)\right]\right\}, \quad j=a, i \\
& \psi_{e}=\frac{1}{4} n_{e} \bar{v}_{e} \exp \left(-e \phi_{f} / k_{B} T_{e}\right)
\end{aligned}
$$

where $\bar{v}_{j}=\sqrt{8 k_{B} T_{j} / \pi m_{j}}$ is the mean thermal velocity of the $j$ th plasma species. $S_{j}=u / \sqrt{2 k_{B} T_{j} / m_{j}}$ is the speed ratio of the relative velocity to the thermal velocity, and $S_{i} \approx S_{a}=S_{h}, S_{e} / S_{h}=\sqrt{m_{e} T_{h} / m_{h} T_{e}} \ll 1$.

The floating potential of the particle can be obtained by balancing the local number flux of ions to that of electrons for nonmetallic particles or by balancing the total number flux of ions to that of electrons over the whole 
particle surface for metallic ones,

$$
\begin{aligned}
\phi_{f}= & \frac{k_{B} T_{e}}{e} \ln \sqrt{\frac{m_{e} T_{h}}{m_{h} T_{e}}}+\frac{k_{B} T_{e}}{e} \\
& \times \ln \left\{\exp \left(-S_{h}^{2} B_{z}^{2}\right)+\sqrt{\pi} S_{h} B_{z}\left[1+\operatorname{erf}\left(S_{h} B_{z}\right)\right]\right\}
\end{aligned}
$$

for nonmetallic particles, and

$$
\begin{aligned}
\phi_{f}^{\prime}= & \frac{k_{B} T_{e}}{e} \ln \sqrt{\frac{m_{e} T_{h}}{m_{h} T_{e}}}+\frac{k_{B} T_{e}}{e} \ln \frac{1}{S_{p}} \iint_{S_{p}} \\
& \times\left\{\exp \left(-S_{h}^{2} B_{z}^{2}\right)+\sqrt{\pi} S_{h} B_{z}\left[1+\operatorname{erf}\left(S_{h} B_{z}\right)\right]\right\} d s
\end{aligned}
$$

for metallic particles, where $d s=a^{2} \sin \theta \sqrt{\cos ^{2} \theta+(c / a)^{2} \sin ^{2} \theta} d \theta d \varphi$ and the surface area of the particle is $S_{p}=\iint_{S_{p}} d s$.

\subsection{Pressure and Shear Stress on the Particle Surface Caused by Plasmas}

The force acting on the particle in the plasma is determined by the momentum exchange between the particle and the plasma. Generally, the momentum exchange between particles and heavy plasma species includes the contributions of those incident on and reflected from the particle surface, thus

$$
\begin{aligned}
p_{j}= & \int_{0}^{\infty} \int_{-\infty}^{\infty} \int_{-\infty}^{\infty}\left(m_{j} v_{z j}\right) v_{z} f_{j}^{-} d v_{x} d v_{y} d v_{z} \\
& +\int_{-\infty}^{0} \int_{-\infty}^{\infty} \int_{-\infty}^{\infty}\left(m_{j} v_{z}\right) v_{z} f_{j}^{+} d v_{x} d v_{y} d v_{z} \\
\tau_{j k}= & \int_{0}^{\infty} \int_{-\infty}^{\infty} \int_{-\infty}^{\infty}\left(m_{j} v_{k}\right) v_{z} f_{j}^{-} d v_{x} d v_{y} d v_{z} \\
& +\int_{-\infty}^{0} \int_{-\infty}^{\infty} \int_{-\infty}^{\infty}\left(m_{j} v_{k}\right) v_{z} f_{j}^{+} d v_{x} d v_{y} d v_{z}
\end{aligned}
$$

where $p$ is the pressure and $\tau$ is the shear stress, $j=a, i$, and $k=x, y$ denotes the direction of the shear stress. The velocity distribution of the reflected atoms can be expressed as

$$
f_{j}^{+}=\frac{\psi_{j}}{2 \pi\left(k_{B} T_{s} / m_{j}\right)^{2}} \exp \left[-\frac{v_{x}^{2}+v_{y}^{2}+v_{z}^{2}}{2 k_{B} T_{s} / m_{j}}\right]
$$




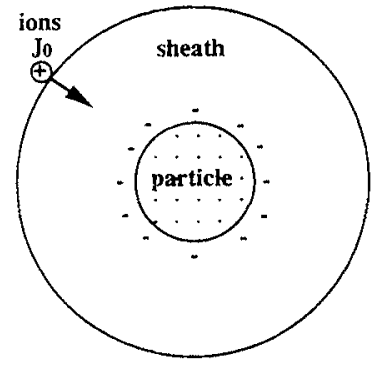

(a)

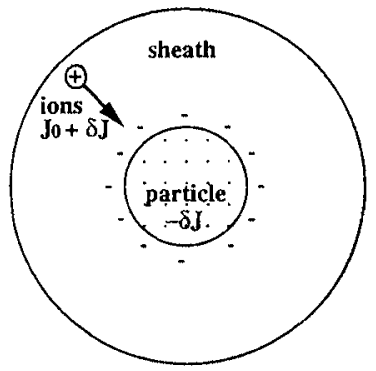

(b)

Fig. 2. Schematic of the moment transfer between particles and ions.

where $T_{s}$ is the particle surface temperature

For electrons, only one part of electrons with velocity $v_{z}>A$ can arrive at the particle surface by overcoming the barrier of floating potential; the other part with velocity $v_{z}<A$ will change their motion direction due to the repulsive action of the electrostatic force within the sheath. Thus the pressure and shear stresses caused by the electrons are

$$
\begin{aligned}
p_{e}= & \int_{A}^{\infty} \int_{-\infty}^{\infty} \int_{-\infty}^{\infty}\left(m_{e} v_{z e}\right) v_{z} f_{e}^{-} d v_{x} d v_{y} d v_{z} \\
& +\int_{0}^{A} \int_{-\infty}^{\infty} \int_{-\infty}^{\infty}\left(2 m_{e} v_{z}\right) V_{z} f_{e}^{-} d v_{x} d v_{y} d v_{z} \\
\tau_{e k}= & m_{e} u_{k} \psi_{e}, \quad k=x, y
\end{aligned}
$$

In the above pressure formula, there is no question concerning $v_{z j}=v_{z}$ for atoms, but different expressions of $v_{z i}$ and $v_{z e}$, corresponding to ions and electrons respectively, were used in the literatures. In Refs. 4, 15, and 16 the use of $v_{z i}=\sqrt{v_{z}^{2}-2 e \phi_{f} / m_{i}}$ and $v_{z e}=\sqrt{v_{z}^{2}+2 e \phi_{f} / m_{e}}$ was suggested. Uglov et $a l .^{(7)}$ suggested using $v_{z j}=v_{z}, j=i, e$. In some early papers, both expressions of $v_{z j}$ were used to study the drag acting on a man-made satellite in outer space; quite different results were obtained in some cases, but no indication was made as to which was reasonable.

According to our opinion, the second expression is correct. A simple analysis is given below.

Consider a negative charging particle in the plasma as shown in Fig. 2 ; for clarity, the sheath around the particle is exaggerated. When the ion is at the sheath edge, as shown in Fig. 2a, the momentum $J_{0}$ of the ion 
which can arrive at the particle is

$$
J_{0}=\int_{0}^{\infty} \int_{-\infty}^{\infty} \int_{-\infty}^{\infty}\left(m_{i} v_{z}\right) v_{z} f_{i}^{-} d v_{x} d v_{y} d v_{=}
$$

After it enters the sheath and strikes the particle, as shown in Fig. 2b, a momentum increment $\delta J$ and a total momentum $J_{0}+\delta J$ is transferred to the particle from the ion, which means the particle obtains momentum $\left(J_{0}+\delta J\right)$ from the ion due to the hitting of the ion. Simultaneously, due to the interaction between the plasma and the particle, a momentum $\delta J$ is produced in the opposite direction in the particle (i.e., $-\delta J$ ). As a result, the net momentum exchange of the particle is

$$
\left(\delta J+J_{0}\right)+(-\delta J)=J_{0}
$$

The same result for the electron can be obtained similarly. This means that for thin sheath conditions the contribution of charged species to the momentum transfer is the same as the neutral species, and it is quite different from the thick sheath conditions ${ }^{(17-19)}$ in the nonequilibrium plasma. Thus we suggest that $v_{z i}=v_{z e}=v_{z}$ be used in the above formula; then the pressure and shear stresses produced by each plasma species are

$$
\begin{gathered}
p_{a}=n_{a} k_{B} T_{h}\left\{\left(\frac{S_{h} B_{z}}{\sqrt{\pi}}+\frac{1}{2} \sqrt{T_{s} / T_{h}}\right) \exp \left(-S_{h}^{2} B_{z}^{2}\right)+\left[1+\operatorname{erf}\left(S_{h} B_{z}\right)\right]\right. \\
\left.\times\left[\frac{1}{2} \sqrt{\pi} \sqrt{T_{s} / T_{h}} S_{h} B_{z}+\left(\frac{1}{2}+S_{h}^{2} B_{z}^{2}\right)\right]\right\} \\
\tau_{a k}=n_{a} k_{B} T_{h} \frac{S_{h} B_{k}}{\sqrt{\pi}}\left\{\exp \left(-S_{h}^{2} B_{z}^{2}\right)+\sqrt{\pi} S_{h} B_{z}\left[1+\operatorname{erf}\left(S_{h} B_{z}\right)\right]\right\}
\end{gathered}
$$

where $k=x, y$,

$$
p_{i}=\alpha p_{a}, \quad \tau_{i k}=\alpha \tau_{a k}
$$

and $\alpha=n_{i} / n_{a}$ is the degree of ionization.

$$
\begin{aligned}
& p_{e}=\frac{2}{\sqrt{\pi}} n_{e} k_{B} T_{e}\left\{S_{e} B_{z} \exp \left(-S_{e}^{2} B_{z}^{2}\right)-\frac{1}{2}\left(C_{f}+S_{e} B_{z}\right) \exp \left[-\left(C_{f}-S_{e} B_{z}\right)^{2}\right]\right. \\
& \left.\quad+\frac{1}{2} \sqrt{\pi}\left(1+2 S_{e}^{2} B_{z}^{2}\right)\left[\frac{1}{2}+\operatorname{erf}\left(S_{e} B_{z}\right)+\frac{1}{2} \operatorname{erf}\left(C_{f}-S_{e} B_{z}\right)\right]\right\} \\
& \tau_{e k}=n_{e} k_{B} T_{e} \frac{S_{e} B_{k}}{\sqrt{\pi}} \exp \left(-C_{f}^{2}\right)
\end{aligned}
$$

where $C_{f}=\sqrt{-e \phi_{f} / k_{B} T_{e}}$ and $k=x, y$. 
Generally, $S_{e} / S_{h} \ll 1$. When $S_{h}$ is not very large, $p_{e}$ and $\tau_{e k}$ can be reduced by setting $S_{e}=0$ :

$$
\begin{aligned}
p_{e} & =n_{e} k_{B} T_{e}\left\{\frac{1}{2}\left[1+\operatorname{erf}\left(C_{f}\right)\right]-C_{f} / \sqrt{\pi} \exp \left(-C_{f}^{2}\right)\right\} \\
\tau_{e x} & =\tau_{e y}=0
\end{aligned}
$$

\subsection{Drag and Rotating Moment}

The components of the force in the direction of the coordinate axes $O X, O Y, O Z$ are

$$
\left(\begin{array}{l}
f_{X_{j}} \\
f_{Y j} \\
f_{Z j}
\end{array}\right)=\left(\begin{array}{ccc}
\cos \alpha & \cos \alpha_{1} & \cos \alpha_{2} \\
\cos \beta & \cos \beta_{1} & \cos \beta_{2} \\
\cos \gamma & \cos \gamma_{1} & \cos \gamma_{2}
\end{array}\right)\left(\begin{array}{c}
p_{j} \\
\tau_{x j} \\
\tau_{y j}
\end{array}\right)
$$

Thus the local drag caused by each plasma species is

$$
f_{D j}=-\left(f_{X j} \sin \theta_{0}+f_{Z j} \cos \theta_{0}\right)
$$

The total drag $F_{D}$ and the drag coefficient $C_{D}$ are defined as

$$
F_{D}=\iint_{S_{p}}\left(f_{D a}+f_{D i}+f_{D e}\right) d s, \quad C_{D}=\frac{F_{D}}{\left(\frac{1}{2} \rho_{\text {plasma }} u^{2}\right) S_{p}}
$$

where $\rho_{\text {plasma }}=m_{a} n_{a}+m_{i} n_{i}+m_{e} n_{e}$ is the plasma density.

The local rotating moment contributed by the three plasma species can be calculated by

$$
\vec{M}=M_{X} \vec{\imath}_{X}+M_{Y} \vec{i}_{Y}+M_{Z} \vec{\imath}_{Z}=\left(\begin{array}{ccc}
\vec{i}_{X} & \vec{i}_{Y} & \vec{\imath}_{Z} \\
X & Y & Z \\
\sum_{j} f_{X_{j}} & \sum_{j} f_{Y_{j}} & \sum_{j} f_{Z_{j}}
\end{array}\right)
$$

where $X, Y, Z$ are the coordinate values of the point on the particle surface; then the moment coefficient is defined traditionally as

$$
C_{m k}=\iint_{S_{p}} M_{k} d s /\left(\frac{1}{2} \rho_{\text {plasma }} u^{2}\right) V_{p}
$$

where $k=X, Y, Z$ denotes the component in the $\mathbf{O X}, \mathbf{O Y}, \mathbf{O Z}$ direction respectively, and $V_{P}=\frac{4}{3} \pi a^{2} c$ is the particle volume. 


\section{RESULTS AND DISCUSSION}

\subsection{Results for Spherical Particles}

For spherical particles, $c=a=R$. The oncoming plasma flow is simply assumed in the direction of $\theta_{0}=0$. Thus $B_{x}=0, B_{y}=\sin \theta$, and $B_{z}=\cos \theta$. Substituting $p_{j}$ and $\tau_{j k}, j=a, i, e$ and $k=x, y$, into Eqs. (10)-(12), we obtain

$$
\begin{aligned}
f_{D j} & =-f_{Z j}=p_{j} \cos \theta+\tau_{y j} \sin \theta \\
F_{D} & =\sum_{j} \int_{0}^{2 \pi} d \varphi \int_{0}^{\pi} f_{D j} R^{2} \sin \theta d \theta \\
& =2 \pi R^{2} \sum_{j} \int_{0}^{\pi}\left(p_{j} \cos \theta+\tau_{y j} \sin \theta\right) \sin \theta d \theta
\end{aligned}
$$

For metallic particles, $\phi_{f}$ is the same on the particle surface; thus, the drag can be expressed analytically:

$$
\begin{aligned}
F_{D}= & 2 \pi R^{2}\left(n_{a}+n_{i}\right) k_{B} T_{h} \\
& \times\left\{\left(S_{h}^{2}+1-\frac{1}{4 S_{h}^{2}}\right) \operatorname{erf}\left(S_{h}\right)+\frac{1+2 S_{h}^{2}}{2 S_{h} \sqrt{\pi}} \exp \left(-S_{h}^{2}\right)+\frac{\sqrt{\pi}}{3} S_{h} \sqrt{T_{s} / T_{h}}\right\} \\
C_{D}= & \frac{1}{2 S_{h}^{2}} \\
& \times\left\{\left(S_{h}^{2}+1-\frac{1}{4 S_{h}^{2}}\right) \operatorname{erf}\left(S_{h}\right)+\frac{1+2 S_{h}^{2}}{2 S_{h} \sqrt{\pi}} \exp \left(-S_{h}^{2}\right)+\frac{\sqrt{\pi}}{3} S_{h} \sqrt{T_{s} / T_{h}}\right\}
\end{aligned}
$$

The contribution of electrons is negligible when $S_{e}$ is small; it is consistent with Ref. 6 . The drag coefficient is only dependent on the relative velocity and the plasma temperature.

For nonmetallic particles, the drag can only be obtained by numerical integration of Eq. (15).

In simple terms, due to the perfect symmetric action of the pressure and the shear stress, the moment acting on the spherical particle, both metallic and nonmetallic, equals zero, i.e.,

$$
M_{X}=M_{Y}=M_{Z}=0
$$

\subsection{Results for Metallic Ellipsoid Particles}

Firstly, the drag and rotating moment for ellipsoid metallic particles with axis ratio $c / a$ from 0.1 to 10 are studied in Ar plasma with $T_{e}=T_{h}$. 
Table I. Calculating Conditions

\begin{tabular}{ll}
\hline Plasma pressure & $500 \mathrm{~Pa}$ \\
Plasma temperature & $8000,12,000 \mathrm{~K}$ \\
Speed ratio & $S_{h}=0-1.5$ \\
Particle surface temperature & $T_{s}=1000 \mathrm{~K}$ \\
Ellipsoid axis ratio & $c / a=0.1-10$ \\
\hline
\end{tabular}

The calculating conditions are shown in Table I, and the corresponding electrons and atoms number density are taken from Ref. 12.

The variation of the drag coefficient and the rotating moment coefficient with the angle of plasma oncoming flow are shown in Fig. $3 \mathrm{a}$ and $3 \mathrm{~b} . C_{D}$ remains constant for spherical particles when $\theta_{0}$ varies from 0 to $90^{\circ}$, but it increases monotonically for prolate particles $(c / a>1)$ and decreases monotonically for oblate ones $(c / a<1)$. Furthermore, there is a critical angle

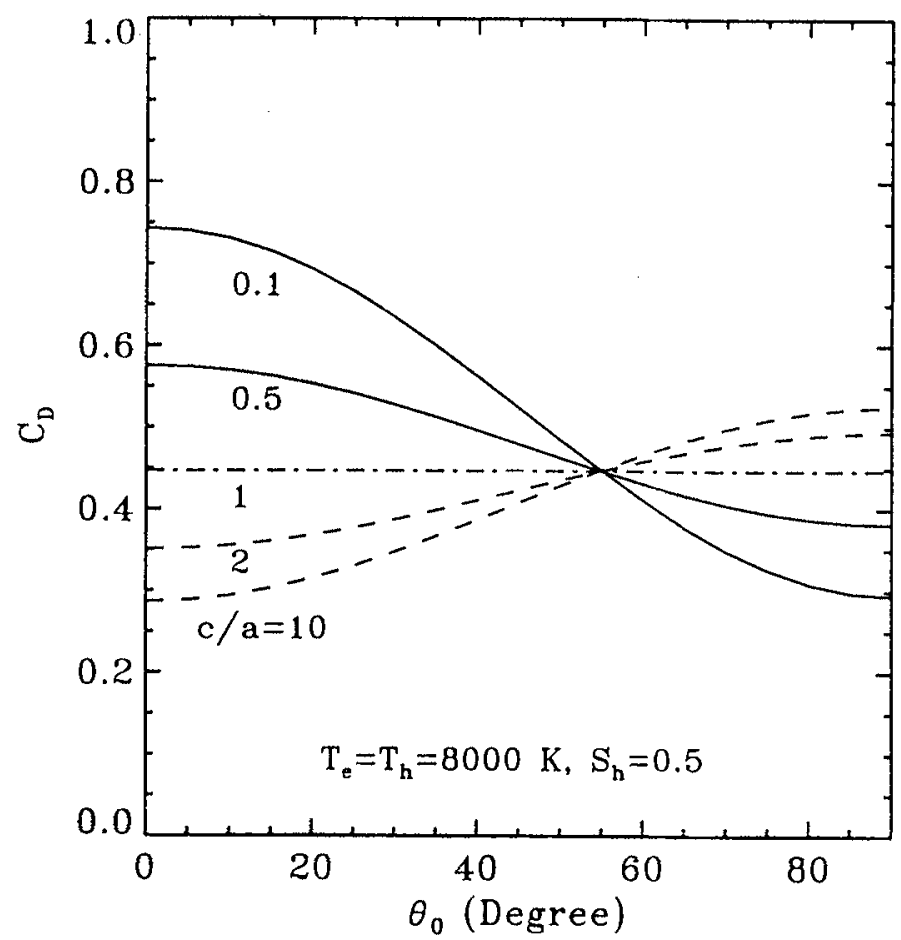

(a)

Fig. 3. Drag coefficient (a) and moment coefficient (b) for ellipsoid particles under the conditions $T_{e}=T_{h}=8000 \mathrm{~K}, S_{h}=0.5$, and $T_{s}=1000 \mathrm{~K}$. 


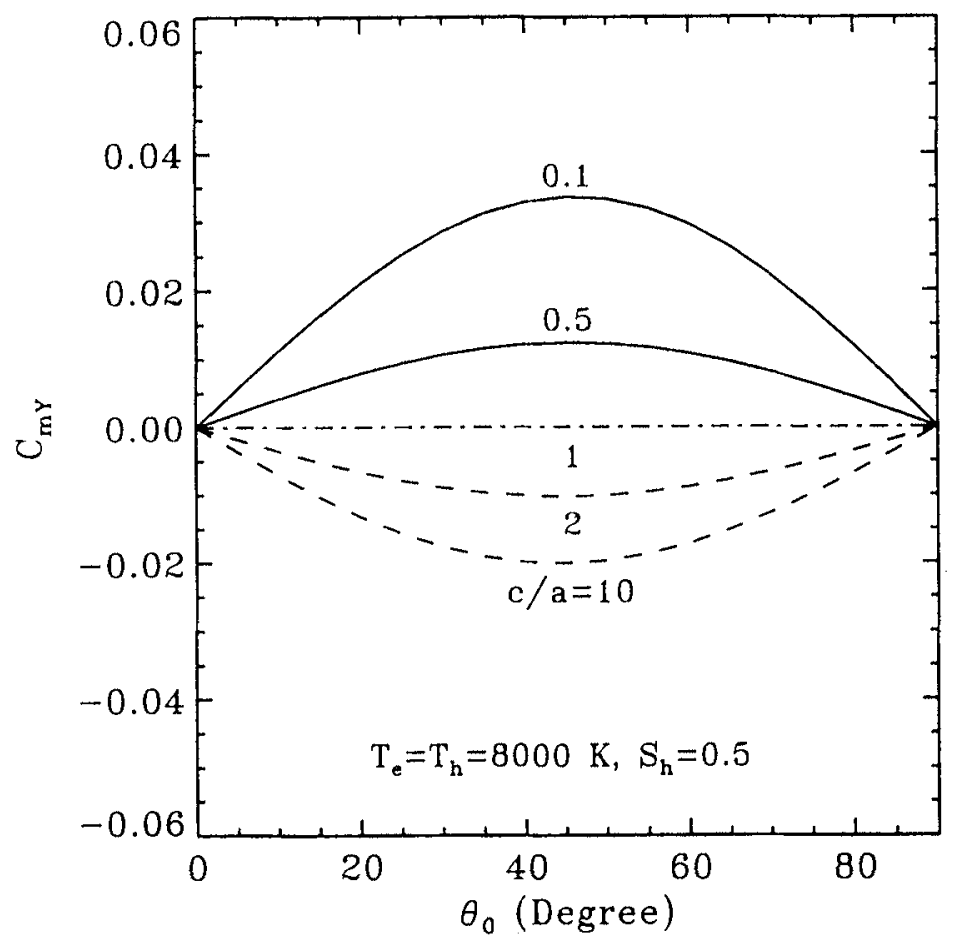

(b)

Fig. 3. Continued.

$\theta_{\text {crit }} \simeq 55^{\circ}$ for an ellipsoid particle of revolution which is independent of $c / a$ and $S_{h}$; when the plasma oncoming flow is in the direction of $\theta_{0}=\theta_{\text {crit }}$ or $\theta_{0}=180-\theta_{\text {crit }}$, the drag of the ellipsoid particles equals that of spherical particles, but it is larger when $\theta_{\text {crit }}<\theta_{0}<180-\theta_{\text {crit }}$ and smaller when $\theta_{0}<\theta_{\text {crit }}$ or $\theta_{0}>180-\theta_{\text {crit }}$ for prolate particles than for spherical ones. The opposite results are obtained for oblate particles. We can also see that larger oscillations of the drag with $\theta_{0}$ can be found for ellipsoid particles when they deviate from much larger spherical particles, i.e., increasing $c / a$ for prolate ellipsoid particles or decreasing $c / a$ for oblate ones will result in a larger oscillation of the drag with the oncoming angle of plasma flow. As to the rotating moment, $M_{X}$ and $M_{Z}$ always equal zero due to the symmetry of local pressure and shear stresses on the ellipsoid particle with respect to the $\mathbf{O X}$ and $\mathrm{OZ}$ axis, but $M_{Y}$, which is similar to the pitching moment defined in the aerodynamics, never equals zero except in two special directions which are parallel to the axis, i.e., $\theta_{0}=0$ or $90^{\circ}$, and it reaches 
the peak value when $\theta_{0}$ is about $45^{\circ}$, as shown in Fig. $3 \mathrm{~b}$. For prolate particles, when $\theta_{0}=0-90^{\circ}, M_{Y}$ is negative, which means that a clockwise

angular acceleration viewed from the direction of $\overrightarrow{Y O}$ should be generated and the particle would be self-rotating or self-oscillating around axis $\mathbf{O Y}$. On the contrary, for oblate particles, positive $M_{Y}$ results in a counterclockwise angular acceleration. Therefore, it may be concluded that any ellipsoid particles (nonspherical particles) in a rarefied plasma would be self-rotating or self-oscillating around its own axis and thus the drag force acting on the nonspherical particles is oscillating correspondingly. Although this point has not been reported, as we know, in plasma experiments or theory analyses the phenomenon of particle self-rotating in many aerosol systems has been observed extensively and is well known to researchers, which can be confirmed in the present analyses by setting $\phi_{f}=0$ and $n_{i}=0$. Thus, we can say that this serves as an indirect but firm support of our analytical results. In plasma conditions, it is also not too difficult to verify this phenomenon experimentally by adopting some similar well-developed technologies as those used in combustion.

In a plasma the self-rotating or self-oscillating particle will experience a time-dependent velocity distribution function for each species, while all the results in the present paper are obtained by the time-independent velocity distribution functions (1) and (5). But in a first-order approximation, the effect of this inconsistency on the results is negligible, because for the small particle with a radius of microns, even though the particle has a large angular velocity, the line speed is still small compared with the plasma characteristic speed.

We know that the moment of inertia around axis $O Y$ is $\frac{1}{5} m\left(a^{2}+c^{2}\right)$, where $m$ is the mass of the particle; then the angular acceleration $a_{\theta Y}$ caused by the rotating moment $M_{Y}$ is

$$
a_{\theta Y}=\frac{5}{2} C_{m y} \cdot \frac{\rho_{\text {plasma }}}{\rho_{\text {particle }}} \cdot \frac{u^{2}}{a^{2}+c^{2}}
$$

For the plasma conditions adopted in the present paper (Ar plasma, $p=$ $500 \mathrm{~Pa}, T_{k z}=2000-12,000 \mathrm{~K}$ ), we have approximately $\rho_{\text {plasma }} / \rho_{\text {paricle }}=10^{-5}$ $10^{-7}, u^{2}=S_{h}^{2} \cdot\left(2 k_{B} T_{h} / m_{h}\right)=\left(10^{6}-10^{7}\right) \cdot S_{h}^{2}(m / s)^{2}, C_{m Y} \sim 0.01$; thus $a_{\theta Y}$ is of the order of magnitude $(0.01-1) \cdot S_{h}^{2} /\left(a^{2}+c^{2}\right) \mathrm{rad} / \mathrm{s}^{2}$. For a particle with size several or tens of microns, which is the typical particle size encountered in thermal plasma processing, the angular acceleration can reach as high as $\left(10^{8}-10^{10}\right) \cdot S_{h}^{2} \mathrm{rad} / \mathrm{s}^{2}$, which means that high-speed self-oscillating or selfrotating takes place and the motion cycle is very short.

The effect of $S_{h}$ on the drag coefficient and rotating moment coefficient of prolate $(c / a=2)$ and oblate $(c / a=0.5)$ particles for different plasma temperatures $T_{e}=T_{h}=8000$ and $12,000 \mathrm{~K}$, which correspond to a low degree of 


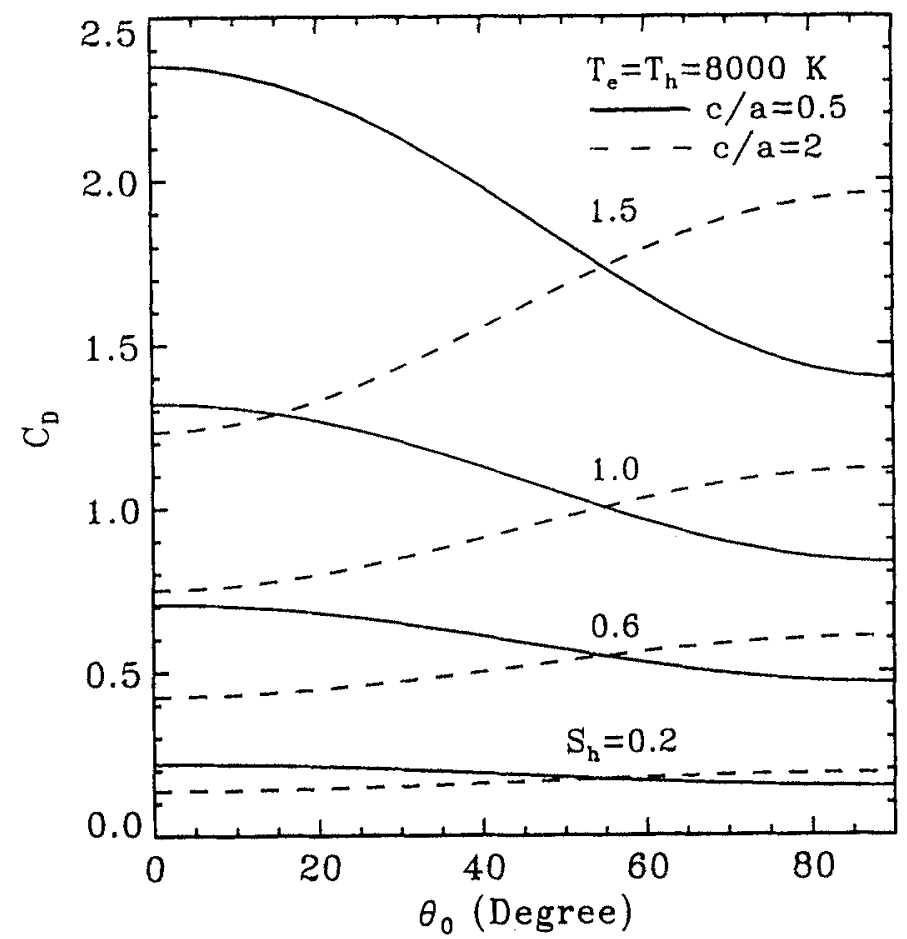

(a)

Fig. 4. Effect of the particle-plasma relative velocity on the drag $(a, b)$ and moment $(c, d)$ coefficients for the prolate and oblate particles under the conditions $T_{e}=T_{h}=8000,12,000 \mathrm{~K}$, $S_{h}=0.2-1.5$, and $T_{s}=1000 \mathrm{~K}$.

ionization $\alpha \simeq 0.023$ for $T_{e}=T_{h}=8000 \mathrm{~K}$ and a high degree of ionization $\alpha \simeq 0.85$ for $T_{e}=T_{b}=12,000 \mathrm{~K}$, is shown in Figs. $4 \mathrm{a}-4 \mathrm{~d}$, respectively. As expected, when $S_{h}=0$ no drag and no rotating moment act on the particles due to the homogeneity of plasma species. For ellipsoid particles when $S_{h}$ increases, both the drag coefficient and the rotating moment coefficient increase greatly. Comparisons of Fig. $4 \mathrm{a}$ with Fig. $4 \mathrm{~b}$ and Fig. $4 \mathrm{c}$ with Fig. $4 \mathrm{~d}$ demonstrate that when the plasma temperature varies, the variations of both the drag and the moment acting on the particle are small, especially for the drag.

\subsection{The Effects of Particle Materials}

The effects of particle materials on the drag coefficient and moment coefficient are also investigated. It is very interesting that when the 


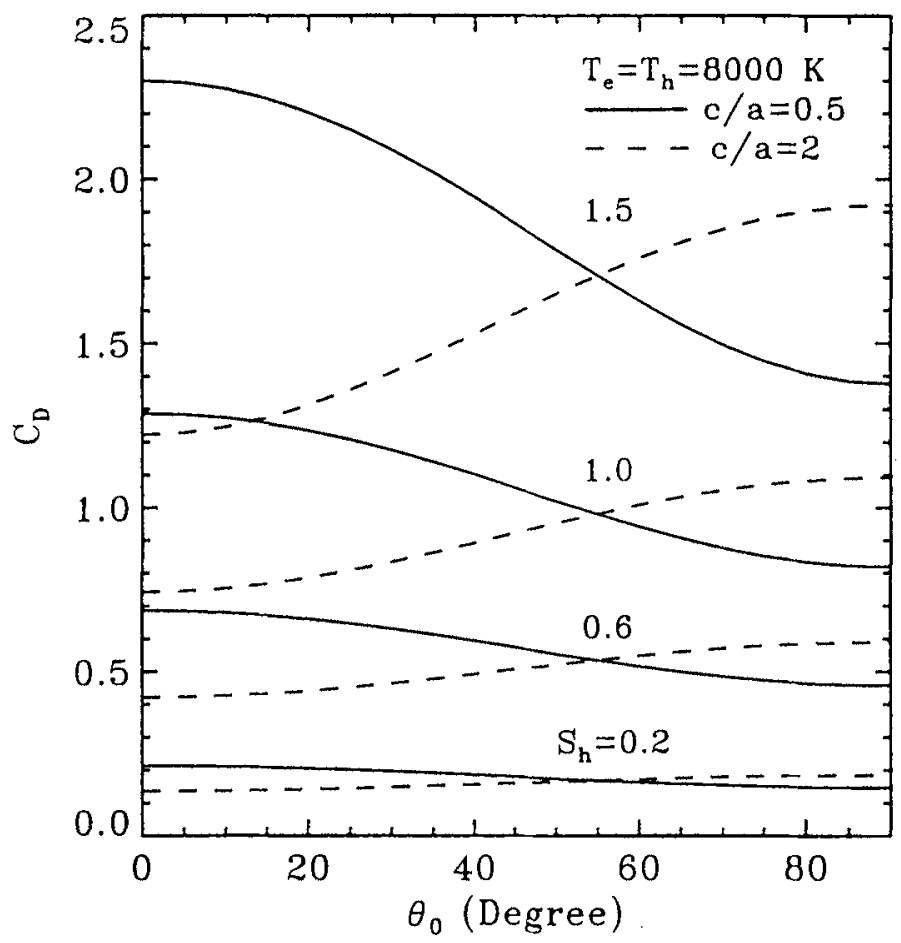

(b)

Fig. 4. Continued.

contribution of electrons on the drag and moment is negligible (it is always negligible because of the small electron mass compared to ions and much smaller $S_{e}$ compared to $S_{h}{ }^{(6.7,15)}$ from Eqs. (6)-(14) we know that the floating potential of the particle is affected by the particle material, metal or nonmetal, but it has no effect on the drag and moment, i.e., the drag and moment acting on the particle are independent of the particle materials.

\subsection{Self-Rotating Frequency of the Particle}

Now, let us estimate the self-rotating frequency of the particles. Assume that the initial angular velocity of the particle is zero, and consider the particle motion within the angle range of 0 to $\pi / 2$; take the average momentum coefficient $\overline{C_{m Y}}$, and then the average angular acceleration $\overline{a_{\theta Y}}$, 


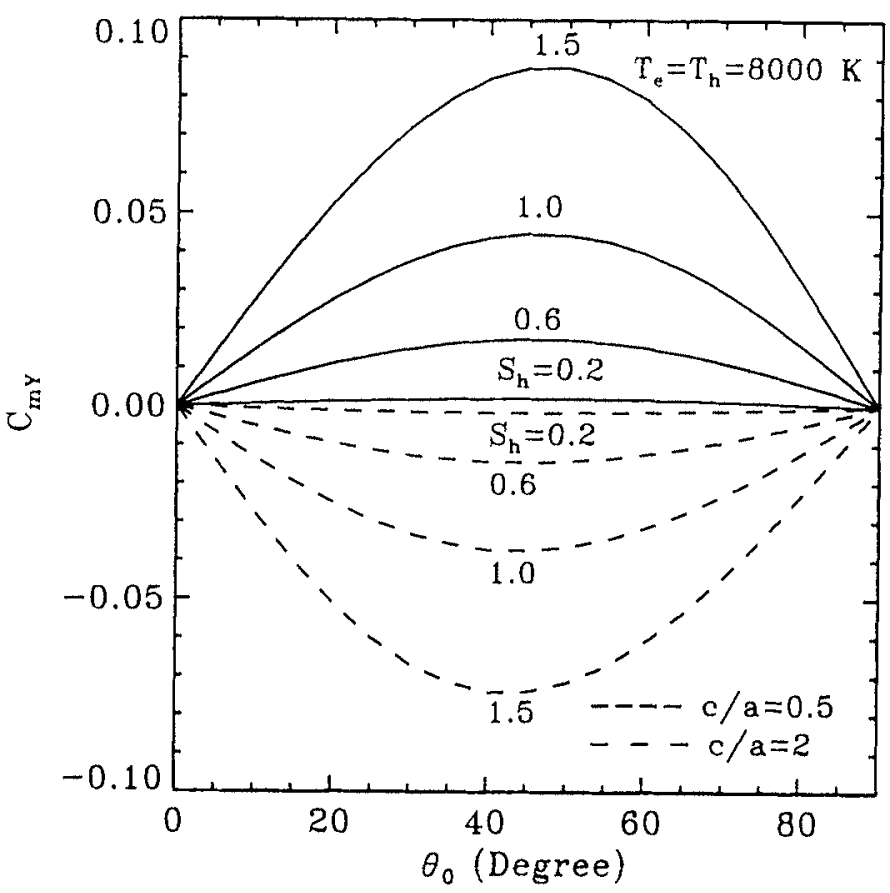

(c)

Fig. 4. Continued.

similar to Eq. (19),

$$
\overline{a_{\theta Y}}=\frac{5}{2} \overline{C_{m y}} \cdot \frac{\rho_{\text {plasma }}}{\rho_{\text {particle }}} \cdot \frac{u^{2}}{a^{2}+c^{2}}
$$

then

$$
\frac{1}{2} \pi=\frac{1}{2} \overline{a_{\theta Y}} \cdot\left(\frac{T}{4}\right)^{2}
$$

where $T$ is the period of particle rotation and $f=1 / T$ is the frequency. It is easy to get

$$
f=\left(\frac{a_{\theta Y}}{\frac{1}{4} \pi}\right)^{1 / 2} \propto \frac{S_{h}}{\left(\rho_{\text {particle }}\right)^{1 / 2} \cdot\left(a^{2}+c^{2}\right)^{1 / 2}}
$$




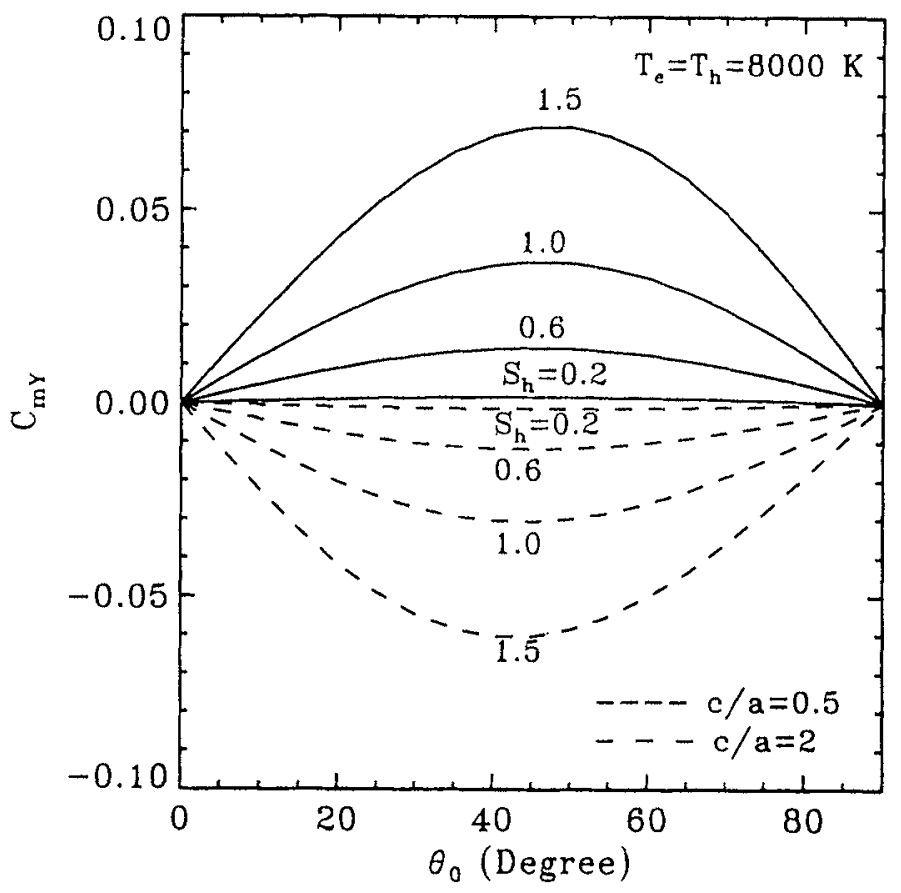

(d)

Fig. 4. Continued.

We can see that $f$ is roughly proportional to the plasma-particle relative velocity and inversely proportional to the particle size and the square root of the particle material density.

\section{CONCLUSION}

Under conditions of free-molecular flow and thin plasma sheath, the following main conclusions are drawn on the drag and rotating moment for ellipsoid particles of revolution:

1. The nonspherical particles in a rarefied plasma flow or in ordinary rarefied flow are always in a self-oscillating or self-rotating state due to the action of the rotating moment, and thus the drag force on the particle is correspondingly oscillating.

2. Both the drag force and the rotating moment increase when the plasma-particle relative velocity increases. 
3. The effect of the plasma temperature on the drag and moment is very small.

4. When the contribution of electrons on the drag and moment is negligible, the drag and moment acting on the particle are materialindependent.

5. The particle self-rotating frequency is inversely proportional to the particle size and the square root of the particle material density and directly proportional to the plasma-particle relative velocity.

\section{ACKNOWLEDGMENTS} of China.

This work was supported by the National Natural Science Foundation

\section{REFERENCES}

1. E. Pfender, Pure Appl. Chem. 60, 591 (1988)

2. M. I. Boulos, IEEE Trans. Plasma Sci. Technol. 19, 725 (1991).

3. T. Honda, T. Hayashi, and A. Kanazama, Int. J. Heat Mass Transfer 24. 1249 (1981).

4. R. Godard and J. S. Chang, J. Phys. D: Appl. Phys. 13, 2005 (1980).

5. R. L. Boxman and S. Goldsmith, J. Appl. Phys. 52, 151 (1981).

6. Xi Chen and Ping He, Plasma Chem. Plasma Process. 6, 313 (1986).

7. A. A. Uglov and A. G. Gnedovets, Plasma Chem. Plasma Process. 11, 251 (1991)

8. A. G. Gnedovets and A. A. Uglov, Plasma Chem. Plasma Process. 12, 371 (1992).

9. Xi Chen. Ji Chen, and Y. D. Yang, J. Phys. D: Appl. Phys, 26, 1637 (1993).

10. Chen Yunming and Li Ming, J. Phys. D: Appl. Phys. 26, 1007 (1993).

11. Li Ming and Chen Yunming, Scientific and Technological Report IMCAS STR-93015, 1993.

12. Li Ming, Chen Yunming, and Hanming Wu, J. Phys. D: Appl. Phys. 27, 1781 (1994).

13. A. G. Gnedovets and A. A. Uglov, Plasma Chem. Plasma Process. 12, 383 (1992)

14. Li Ming and Chen Yunming, IEEE Trans. Plasma Sci. Technol. 22, 449 (1994).

15. Xi Chen and Xizoming Chen, Plasma Chem. Plasma Process. 9, 387 (1989).

16. Xi Chen, Bingzhi Su, and Yu Lan, Plasma Chem. Plasma Process. 15, I (1995).

17. M. D. Kilgore, J. E. Daugherty, R. K. Porteous, and D. B. Graves, J. Appl. Phys. 73, $7195(1993)$

18. M. S. Barnes, J. H. Keller, J. C. Forster, J. A. O'Neill, and D. K. Coultas, Phys. Rev. Lett. 68, 313 (1992)

19. G. S. Selwyn, J. Singh, and R. S. Bennett, J. Vac. Sci. Technol. A 7, 2758 (1991). 